\title{
Mappings into normed linear spaces
}

\author{
by \\ V. Kle e * (Copenhagen and Seattle)
}

We contribute a few new fragments to a still fragmentary theorythat of the topological structure of infinite-dimensional normed linear spaces. $\$ 1$ is concerned with a problem of Fréchet [6] and Banach [1]: Are all infinite-dimensional separable Banach spaces homeomorphic? Kadeč $[7,8]$ recextly obtained an affirmative answer for the case of reflexive spaces. With the aid of a mapping theorem of Whyburn [29], we are able to extend the reasoning of [8] to cover all infinite-dimensional separable conjugate spaces. $\S 2$ begins with some remarks on linear transformations of spaces ls, extending a result of Banach and Mazur [2]. In conjunction. with a theorem of Bartle and Graves [3], this leads to some interesting corollaries such as an embedding theorem of Dowker [5] and the fact that every metric space of cardinality $\leqslant c$ admits a biunique continuous map onto some totally bounded metric space ( $\left.{ }^{3}\right)$. An example in $\$ 2$ substantiates a conjecture in Michael's selection theory [24]. A few other results are obtained and some unsolved problems are stated.

\$ 1. The theorem of Kadeč. A subset $X$ of a metric space will be called a Tehebycheff set provided each point of the space admits a inique nearest point in $X$. An admissible norm for a normed linear space is one which generates the same topology as the given norm.

Kadeč first proved [7] that all infinite-dimensional separable uniformly convex Banach spaces are homeomorphic, then later observed [8] that the relevant consequences of uniform convexity can be obtained in more general spaces. By careful analysis of his reasoning, one arrives at the following conclusion.

1.1. THEORm (Kadeč). Two infinite-dimensional normed linear spaces $E_{1}$ and $E_{2}$ are homeomorphic if $($ for $i=1,2)$ there exist an admissible norm \|\| for $E_{i}$, a linear subspace $F_{i}$ of the conjugate space $E_{i}^{*}$, and a linearly independent sequence $f_{a}$ in $F_{i}$ such that the following three conditions are satisfied:

* Research fellow of the Alfred P. Sloan Foundation.

( $)$ Added in proof: A simpler proof of this fact has been communicated to the author by Professor H. H. Corson. 
$1^{0}$ the unit cell $\left\{x \in E_{i}:\|x\| \leqslant \mathcal{X}\right\}$ is compact in the topology $\sigma\left(E_{i}, F_{i}\right)$; $2^{0}$ with $L_{n}=\left\{x \in E_{i}: 0=f_{1} x=\ldots=f_{n} x\right\}, \bigcap_{1}^{\infty} L_{n}=\{0\}$ and each $L_{n}$ is a Tchebycheff set in $E_{i}$;

$3^{0}$ if $x_{\sigma}$ is a sequence in $E_{i}, x \in E_{i},\left\|x_{\alpha}\right\| \rightarrow\|x\|$, and $f x_{\alpha} \rightarrow f x$ for all $f \in F_{i}$, then $\left\|x_{a}-x\right\| \rightarrow 0$.

The following corollary of 1.1 may be of interest in connection. with the problem of Fréchet and Banach. Notice that a priori it does not seem obrious even that $E$ is separable.

1.2. ConollaRY. Suppose that $E$ is an infinite-dimensional linear subspace of $R^{\mathrm{N}_{0}}$, topologized by means of a norm which satisfies the following two conditions:

$1^{0}$ the unit cell $\left\{x=\left(x^{1}, x^{2}, \ldots\right) \in E:\|x\| \leqslant 1\right\}$ is compact in the topology of pointwise convergence;

$2^{\circ}$ if $x_{\alpha}$ is a sequence in $E, x \in E,\left\|x_{a}\right\| \rightarrow\|x\|$, and $x_{a}^{n} \rightarrow x^{n}$ for each $n$, then $\left\|x_{a}-x\right\| \rightarrow 0$.

Then the space $E$ is homeomorphic with Hilbert space.

Proof. It is evident that Hilbert space satisfies the conditions of 1.1. We wish to prove the same for the space $E$. For $n=1,2, \ldots$, let the linear functional $g_{n}$ on $E$ be defined as follows: $g_{n}\left(x^{1}, x^{2}, \ldots\right)=x^{n}$. From $1^{0}$ it follows that $\left.\sup \mid g_{n} x\right\}=B_{n}<\infty$, whence $g_{n}$ is continuous for the norm topology. Luet $F$ denote the linear extension of $\left\{g_{n}\right\}_{1}^{\infty}$, so that $F \subset E^{*}$. For each $x \in E$, let

$$
(x)=\left[\sum_{1}^{\infty}\left(2^{-n / 2} x_{n} / B_{n}\right)^{2}\right]^{1 / 2} .
$$

Then () is a norm for $E$ and ()$\leqslant\|\|$, so the function $(())$, with

$$
((x))=\|x\|+(x),
$$

is an admissible norm for $E$. It can be verified that the unit cell $\{x \in E:(x) \leqslant 1\}$ is compact in the topology of pointwise convergence and that if $x_{\alpha}$ is a sequence in $E, \infty \in E,\left\|x_{\alpha}\right\|$ is bounded, and $x_{a}^{n} \rightarrow x^{n}$ for each $n$, then $\left(x_{a}-x\right) \rightarrow 0$.

Since both || || and ( ) satisfy condition $1^{\circ}$, it follows that both are lower semicontinuous for the topology of pointwise convergence, whence $(())$ is lower semicontinnous and satisfies condition $1^{\circ}$. Consequently, the unit cell $\{x \in \mathbb{E}:((x)) \leqslant 1\}$ is compact in the topalogy $\sigma(E, F)$. Now consider a sequence $x_{a}$ in $E$ and a point $x \in E$ such that $\left(\left(x_{a}\right)\right) \rightarrow[(x))$ and $f x_{c} \rightarrow f x$ for all $f \in \mathbb{F}$. Then of course $\left\|x_{a}\right\|+\left(x_{a}\right) \rightarrow\|x\|+(x)$, and $x_{\alpha}^{n} \rightarrow x^{n}$ for all $n$. Clearly $\left\|x_{\alpha}\right\|$ is bounded and consequently $\left(x_{\alpha}-x\right) \rightarrow 0$, whence $\left\langle x_{\mathrm{a}}\right) \rightarrow(x)$ and $\left\|x_{a}\right\| \rightarrow\|x\|$. Thus from $2^{\circ}$ it follows that $\left\|x_{\alpha}-x\right\| \rightarrow 0$ and hence $\left(\left(x_{\alpha}-x\right)\right) \rightarrow 0$. We have proved that the triple $(E,(()), F)$ satisfies conditions $1^{\circ}$ and $3^{\circ}$ of 1.1 .

Now it is easy to produce a subsequence $f_{\alpha}$ of $g_{\alpha}$ such that $f_{\alpha}$ is linearly independent over $E$ and such that if $L_{n}=\left\{x \in E: 0=f_{1} x=\ldots=f_{n} x\right\}$, then $\bigcap_{1}^{\infty} L_{n}=\{0\}$. To complete the proof it remains only to show that each $L_{n}$ is a Tchebycheff set in $E$. Consider an arbitrary point $y \in E$ and let $U=\{x \in E:((x)) \leqslant 1\}$. From compactness of $U$ (in the topology of pointwise convergence) and the special form of $L_{n}$, it follows that the set $(y+t U) \cap L_{n}$ is also compact for every $t \geqslant 0$. Thus there exists a smallest value $t_{0}$ of $t$ among those for which the intersection is nonempty. But it is easy to verify that the norm ( $)$ is strictly convex, whence (( )) is also strictly convex and the set $\{x \in E:((x))=1\}$ cannot contain any line segment. Consequently the set $\left(y+t_{0} U\right) \cap L_{n}$ consists of a single point of $L_{n}$, and the set $L_{n}$ must be a Tchebycheff set. It now follows from 1.1 that $E$ is homeomorphic with Hilbert space.

Although Kadeč considers only pointwise convergence over the entire interval $[0,1]$, his reasoning in [8] actually establishes the following result:

1.3. THEOREM (Kadeč). In the space $C[0,1]$, let the norm \|\| be defined as follows:

$$
\|\varphi\|=\max _{t \in[0,1]}|\varphi t|+\left[\int_{0}^{1} \varphi^{2}\right]^{1 / 2}+\sum_{k=1}^{\infty} 2^{-k} \max _{|r-s| \leqslant 1 / k}|\varphi r-\varphi s| .
$$

Then \|\| is a strictly convex admissible norm for $C[0,1]$ which satisfies the following two conditions:

$\mathrm{K}^{1}$-for each set $M$ of measure 1 on $[0,1]$, the unit cell $\{\varphi \in C[0,1]:\|\varphi\|$ $\leqslant 1\}$ is closed $($ in $C[0,1])$ in the topology of pointwise convergence on $M$;

$K^{2}-i f M$ is a set of measure 1 on $[0,1], \varphi_{a}$ is a sequence in $O[0,1]$, $\varphi \in C[0,1],\left\|\varphi_{\alpha}\right\| \rightarrow\left\|\varphi^{\prime}\right\|$, and $\varphi_{a} t \rightarrow \varphi t$ for each $t \in M$, then $\left\|\varphi_{a}-\varphi\right\| \rightarrow 0$.

Since ([1]) every separable Banach space is linearly homeomorphic with a linear subspace of $C[0,1]$, there results

1.4. Corollarry (Kadeč). Every separable Banach space admits a strictly convex norm \|\| such that whenever $x_{\alpha}$ is a sequence in $E, x \in E$, $\left\|x_{a}\right\| \rightarrow\|x\|$, and $x_{a}$ is weakly convergent to $x$, then $\left\|x_{a}-x\right\| \rightarrow 0$.

From 1.1 and 1.4 it follows that all infinite-dimensional separable reflexive Banach spaces are homeomorphic. In order to sharpen that result, we derive the following consequence of 1.3 .

1.5: CoRoltaRY. If $E$ is a Banach space whose conjugate space $E^{*}$ is separable, then there is an admissible norm for $E$ such that the corresponding norm in $E^{*}$ is striotly convex and such that whenever $f_{a}$ is a sequence in $E^{*}$, $f \in \mathbb{B}^{*},\left\|f_{\alpha}\right\| \rightarrow\|f\|$, and $f_{\alpha}$ is $w^{*}$-convergent to $f$, then $\left\|f_{\alpha}-f\right\| \rightarrow 0$. 
Proof. Let $D$ be the set of all dyadic rationals in $[0,1]$. By a wellknown type of construction, there exists a set $M$ of measure 1 in $[0,1]$ and a continuous map $\zeta$ of $[0,1]$ onto itself such that $\zeta M=D$. (See, for example, $[21]$, p. 48-50.) Let $U$ denote the unit cell of $E, U^{* *}$ that of the second conjugate space $E^{* *}$, and $\tau$ the canonical embedding of $E$ into $E^{* *}$. Then $\tau U$ is a $w^{*}$-dense subset of the $w^{*}$-compact convex set $U^{* *}$. Since $E^{*}$ is separable, the space $\left(U^{* *}, w^{*}\right)$ must be homeomorphic with the Hilbert parallelotope $\left([10]\right.$, p. 31). In particular, the space $\left(U^{* * *}, w^{*}\right)$ is a Peano continuum, and by a mapping theorem of Whyburn ([29], p. 31) there exists a continuous map $\eta$ of $[0,1]$ onto $\left(U^{* *}, w^{*}\right)$ such that $\eta D \subset \tau U$.

For each $f \in E^{*}$, let $\mu f$ be the function in $C[0,1]$ defined as lollows:

$$
(\mu f) t=(\eta \zeta t) f
$$

where of course $t \in[0,1]$ and $\eta \xi t \in U^{* * *}$. Then $\mu$ is a linear homeomorphism of $E^{*}$ into $O[0,1]$ under which the $w^{*}$ topology in $E^{*}$ corresponds to the topology in $O[0,1]$ given by pointwise convergence on the set $\zeta^{-1} \eta^{-1}(\tau U)$ $\supset M$. For each $f \in E^{*}$, define $\|f\|=\|\mu f\|$, where the second \|\| is Kades's norm for $C[0,1]$. Property $K^{1}$ in 1.3 implies that the unit cell $\left\{f \in E^{*}\right.$ : $\|f\| \leqslant I\}$ is $w^{*}$ - closed, whence the norm for $E^{*}$ is generated by an admissible norm for $E$. The other properties claimed in 1.5 follow at once from the corresponding properties of Kadee's norm.

We may now establish the principai result of the present section.

1.6. THeorem. Suppose that $S$ is a normed linear space and $Z$ is an infinite-dimensional separable linear subspace of $S^{*}$ which is olosed th the $w^{*}$-topology. Then $Z$ is homeomorphic with Hilbert space.

Proof. Let $z_{a}$ be a sequence dense in $Z$, and for each $j$ let $w_{i t}^{i}$ be a sequence in the unit cell of $S$ for which $\lim z_{j}\left(w_{i}^{j}\right)=\left\|z_{j}\right\|$. Let $F$ denote the closed linear extension of the set of all points $w_{i}^{i}$ and let $E$ denote the subset of $F^{*}$ consisting of all $x \in F^{*}$ such that $x$ is the restriction to $F$ of some member of $Z$. Then $E$ is isometric with $Z$ and is a $w^{*}$-closed subspace of $F^{*}$. Clearly there exists a sequence $f_{a}$ in $F$ which is linearly independent over $E$ and such that $\bigcap_{1}^{\infty}\left\{x \in E: f_{n} x=0\right\}=\{0\}$, where we make the usual identification of $F$ with a subspace of $E^{*}$. Now let $F^{\prime}$ be given the sort of norm described (for $E$ ) in 1.5 and let $(())$ be the corresponding norm for $F^{*}$. It is easy to verify that conditions $1^{\circ}-3^{\circ}$ of. 1.1 are satisfied by the space $E$, the norm $(())$, the subspace $F$ of $E^{*}$, and the sequence $f_{a}$ in $F$. Thus the desired conclusion follows from 1.1.

1.7. CoRoLLARY. All infinite dimensional separable conjugate spaces are homeomorphic.
§ 2. Linear mappings of $l$. The algebraic dimension and density character of a normed linear space $E$ will be denoted by $d_{E}$ and $\delta_{E}$ respectively. It is well-known that card $E=d_{E}$ when $d_{E} \geqslant c$, card $E=c$ when $1 \leqslant d_{L} \leqslant \mathrm{c}, d_{E} \geqslant c$ when $d_{E} \geqslant \kappa_{0}$ and $E$ is complete, and $\delta_{E} \leqslant d_{L}$ when $d_{T} \geqslant \kappa_{0}$. (See, for example, [17] and [18], p. 159.) For a set $Z$, we denote by $l Z$ the linear space of all real-valued functions $\varphi$ on $Z$ for which $\|p\|<\infty$, where $\|p\|=\sum_{z \in Z}|\varphi z| ; \quad l_{F} Z$ is the linear subspace of $l Z$ consisting of all $q \in l Z$ such that $\phi z=0$ for all but finitely many $z \in Z$. For a cardinal number $s, l s$ will denote (somewhat ambiguously) a space $7 . Z$ such that card $Z=s$, and $l_{F} s$ the corresponding subspace. Spaces $l^{2} \mathrm{~s}$ and $l_{F}^{2} \mathrm{~s}$ are similarly defined.

Banach and Mazur observed that every separable Banach space is a continuous linear image of the space $l \kappa_{0}$ ([2], p. 111). We may extend this result as follows:

2.1. Proposition. Suppose that $E$ is a normed linear space of dimension $d_{E} \geqslant \kappa_{0}$ and density character $\delta_{E}$. Then for the existence of a continuous linear transformation whieh-

carries $l_{F}$ s biuniquely onto $E$, it is necessary and sufficient that $\mathrm{k}=d_{E}$;

is open and carries $l_{F} \times$ onto $E$, it is sufficient that $\mathrm{s} \geqslant \operatorname{card} E$;

is open and carries $\mathrm{l}$ onto $E$ it is necessary that $\mathrm{N} \geqslant \delta_{t}$, (when $E$ is complete, this is also sufficient);

carries $l$ biuniquely onto a dense subspace of $E$, it is sufficient that $E$ is complete, $\delta_{E}=\mathrm{s}_{0}$, and $\mathrm{s}_{0} \leqslant \mathrm{~s} \leqslant \mathrm{c}$.

Proof. Let $Z$ be a set of cardinality $s$. For all $z \in Z$ and $z^{\prime} \in Z \sim\{z\}$, set $\phi_{z} z=1$ and $\varphi_{z} z^{\prime}=0$. Then every map $g$ of the set $\left\{\varphi_{z}: z \in Z\right\}$ onto a bounded subset $B$ of $E$ generates a continuous lineas transformation $T_{g}$ of the space $l_{F} Z$ onto the linear extension of $B$. (Continuity of $T_{g}$ follows from subadditivity and positive homogeneity of the norm in $D$, in conjunction with the special form of the norm in $l Z$.) For the first assertion above, let $B$ be a bounded Hamel basis for $E$ and let $g$ be biunique. For the second, let $B$ be the unit cell of $E$. For the third, let $B$ be a dense subset of the rnit cell in $E$, with card $B \leqslant \kappa$, and observe that when $D$ is complete the transformation $T_{g}$ admits a continuous linear extension $u$ to $l Z$. As in [2], it can be verified that $u(l Z)=E$. That $u$ is open can be verified directly or deduced from the open mapping theorem [1].

Under the hypotheses for the fourth assertion, $E$ is an infiniterimensional separable Banach space. From [12], p. 193, it follows that $W$ dimensional separable Banach space. linear extension is dense in $E$ and such that whenever $a_{a}$ and $b_{\alpha}$ are sequences of real numbers with $\sum_{i}^{\infty} a_{i} x_{i}=\sum_{1}^{\infty} b_{i} x_{i}$, then $a_{j}=b_{j}$ for all $j$. Let $U^{*}$ denote the unit cell of the conjugate space $(l Z)^{*}$. Then in the 
usual way, $U$ may be identified with the set of all real-valued functions $\xi$ on $Z$ for which $\sup _{z \in Z}|\xi \alpha| \leqslant 1$-that is, with the product space $[-1,1]^{Z}$. It is easily verified that the $w^{*}$-topology for $U$ coincides with the produet topology. Since $s \leqslant c$, a theorem of Marczewslzi ([19], p. 139) implies the existence of a countable sequence $f_{a}$ which is $w^{*}$-dense in $U$. Olearly $\bigcap_{1}^{\infty} f_{i}^{-1} 0=\{0\}$, and $f_{a}$ admits a linearly independent subsequence $g_{a}$ such that $\bigcap_{1}^{\infty} g_{i}^{-1} 0=\{0\}$. For each $\varphi \in l Z$, let

$$
T \varphi=\sum_{1}^{\infty} 2^{-n}\left(g_{n} \varphi\right) x_{n} \in E .
$$

If $\sup _{n}\left\|\infty_{n}\right\|=M<\infty$, it can be verified that $\|T \varphi\| \leqslant M\|\varphi\|$, and hence $T$ is continuous. Since the sequence $g_{a}$ is linearly independent, it follows that $T(Z Z)$ contains the linear extension of $\left\{x_{n}\right\}_{1}^{\infty}$, hence is dense in $E$. And the strong linear independence of $x_{\alpha}$ (from the definition of quasibasis) implies that $T$ is biunique. The proof of 2.1 is complete.

We next state a consequence of a theorem of Bartle and Graves ([3], p. 404). It was proved also by Michael $[22,23]$.

2.2. THEOREM (Bartle-Graves). If $E$ and $F$ are Banach spaces and $u$ is a continuous linear transformation of $F$ onto $E$, then there exists a continuous map $v$ of $E$ into $F$ such that uvx $=x$ for every $x \in \mathbb{E}$.

2.3. Conollary. Let $G$ denote the kernel $u^{-1} 0$ of $u$, and for each $y \in F^{r}$ let $h y=(u y, v u y-y) \in E \times G$. Then $h$ is a homeomorphism of $F$ onto $Z \times G$.

The proof of 2.2 is based on the fact that every metric space is paracompact (A. H. Stone [28]). From 2.1 and 2.3 we obtain a new proof of an embedding theorem of Dowker ([5], p. 939), whose proof used. paracompactness directly. While the proof is more complicated than Dowker's, it may nevertheless be of interest.

2.4. CoRollari (Dowker). If a metric space is of density character $\leqslant s$, then it can be topologically embedded in the space $l s$.

Proof. It suffices to consider the case $s \geqslant \kappa_{0}$. Consider a metric space $(M, \varrho)$ having a dense subset $D$ of cardinality $\leqslant \mathrm{k}$. Choose $q_{0} \in M$, and for each $q \in M$ let the real-valued function $\varphi_{q}$ on $M$ be defined as follows:

$$
\varphi_{q} p=\varrho(p, q)-\varrho\left(p, q_{0}\right) \quad(p \in M) .
$$

For each $q \in M$, let $\tau q=\varphi_{q} \in C M$ (the space of all bounded continuous real-valued functions on $M$, with $\left.\|\xi\|=\sup _{x \in M}|\xi x|\right)$. Then $\tau$ is an isometric embedding of $(M, \varrho)$ into $C M$, due essentially to Kuratowski ([16], p. 543). Let $L$ denote the rational linear extension of the set $\tau D$, and let $D$ denote the closure of $L$. Then card $L \leqslant N$ and $E$ is a complete linear subspace of $C M$, so it follows by 2.1 that the space $l s$ admits a continuous linear transformation onto $E$. The desired conclusion now follows from 2.3 .

Dowker actually embedded in $l^{2} \times$ rather than $l s$, but the two spaces are homeomorphic under Mazur's mapping [20] which sends the function $\phi \in l^{2} \times$ into the function $(\operatorname{sgn} \varphi) p^{2} \in l$ s.

2.5. CoRollaky. For a metric space $X$, the following three assertions are equivalent:

$1^{0} X$ is of cardinatity $\leqslant c$

$2^{\circ} X$ admits a biunique continuous map into a compact metric space (that is, onto a totally bounded metric space);

$3^{0}$ there is a sequence $G_{\alpha}$ of open subsets of $X$ such that whenever $p$ and $q$ are distinct points of $X$, then for some $i, p \in G_{i} \subset X \sim\{q\}$.

Proof. It is easily verified that $2^{\circ}$ implies $1^{\circ}$ and $2^{\circ}$ implies $3^{\circ}$. To prove that $1^{\circ}$ implies $2^{\circ}$ it suffices, in view of 2.4 , to show that the space lo admits a biunique continuous map into a compact metric space. But by 2.1, to must admit a biunique continuous map into Hilbert space $l^{2} \kappa_{0}$, and of course Hilbert space is homeomorphic with a subset of the (compact) Hilbert parallelotope. It remains only to show that $3^{\circ}$ implies $2^{\circ}$

Let $\varrho$ be a bounded metric for $X$ and let $G_{\alpha}$ be a sequence of open sets as described in $3^{\circ}$. For each $x \in X$ and each positive integer $n$, let

$$
f_{n} x=\frac{1}{n} \inf _{y \in X \sim G_{n}} \varrho(x, y) \text {. }
$$

Then set $\varphi x=\left(f_{1} x, f_{2} x, \ldots\right) \in l^{2} \kappa_{0}$. Clearly $\varphi$ is biunique. It is easy to see that $\varphi$ is continuous and $\varphi X$ lies in a compact subset of $l^{2} \kappa_{0}$. The proof of 2.5 is complete.

Parhomenko [25] has treated some related problems, characterizing topological spaces which admits biunique continuous maps onto certain types of spaces. Still open is Banach's problem of characterizing those metric spaces, and especially those Banach spaces, which admit a biunique continuous map onto some compact metric space. Aspects of his problem have been treated in [9], [11], [13], [27]. It is known that the space $\left(c_{0}\right)$, every separable conjugate space, and the space $(m)$ all admit biunique continuous maps onto the Filbert parallelotope. Does the space lo admit such a map? Does $l_{e}$ admit a homeomorphism into $(m)$ or a biunique continuous map onto $l_{0}$ ? It can be seen that $l_{F} c$ admits a biunique continuous map onto a compact metric space, while $l_{F} k_{0}$ does not.

Now consider a set $S$ and the corresponding product space $S^{n}$, where $n$ is a positive integer. In [12] we defined a cross-section of $S^{n}$ to be a subset $C_{n}$ of $S^{n}$ such that if $\left(x_{1}, \ldots, x_{n}\right) \in C_{n}$, then the $x_{i}$ 's are all distinct, and 
such that whenever $y_{1}, \ldots, y_{n}$ are $n$ distinct points of $S$, there is exactly one permutation $z_{1}, \ldots, z_{n}$ of the $y_{i}^{\prime}$ 's for which $\left(z_{1}, \ldots, z_{n}\right) \in O_{n}$.

2.6. Coroldarx. If $S$ is a metric space of cardinality $\leqslant \mathrm{c}$, then $\mathrm{s}^{\prime \prime}$ admits a cross-section which is the whion of a countable family of closed sets.

Proof. Let $\xi$ be a biunique continuous map of $S$ into a compact metric space $K$, and for $\left(x_{1}, \ldots, x_{n}\right) \in S^{n}$ define $\eta\left(x_{1}, \ldots, x_{n}\right)=\left(\xi x_{1}, \ldots, \xi x_{n}\right)$ $\epsilon K^{n}$. By [12], p. 191, there is an $F_{\sigma}$ cross-section $G_{n}$ for $K^{n}$, and then the set $\eta^{-1}\left(C_{n} \cap \eta S^{n}\right)$ is an $F_{\text {to }}$ cross-section for $s^{n^{n}}$.

Now from 2.6 and [12], p. 196, we deduce

3.7. CoRoluary. Suppose that $E$ is a normed linear space of otrdinality $\leqslant c$ (or, equivalently, of dimension $\leqslant c$ ). Then in the whit sphere $\{x \in E$ : $\|x\|=1\}$ there is a countable sequence of closed sets whose nnion includes exactly one point from each antipodal pair.

Corollary 2.7 should be compared with the antipodal point theorems of Lyusternik and Schnirelmann, and Borsuk [4]. I have no satisfactory results concerning extension of 2.6 and 2.7 to spaces of higher cardinality. With the aid of 2.6, some of the results of [12] can be extended. In particular, ,separable metric spaces" may be replaced by "metric spaces of cardinality $\leqslant c$ " in 2.1 of [12].

We wish, finally, to apply 2.1 to prove a conjecture of Michael [24]. For this purpose, we introduce two additional definitions. A separable metric space is strongly infinite-dimensional provided each of its nonempty open subsets is infinite-dimensional. A normed linear space $D$ is accessible provided it admits a Hamel basis $B$ such that whenever $J \subset B$ and card $J<\operatorname{card} B$, then the linear extension of $J$ is closed in $E$. Clearly every $\kappa_{0}$-dimensional normed linear space is accessible, as is every space $l_{F} \leqslant$.

2.8. Proposition. Suppose that $X$ is a separable metric space whioh is strongly infinite-dimensional and $Y$ is a closed subset of $X$ which can be trpologically embedded in an accessible nowed lineain space. Then $Y$ is of the first category in $X$.

Proof. The statement is obvious for all finite-dimensional normed linear spaces. Suppose it is known for all spaces of dimension $<\mathrm{k}$, and consider an $\aleph$-dimensional normed linear space $E$ with Hamel basis $B$. Let $B$ be well-ordered by a reflexive relation $\rightarrow$ such that for each $b \in B$, the set $P_{b}=\{d \in B: d-3 b\}$ is of cardinality $<x$. Consider a strongly infinite-dimensional separable metric space $X$, a closed subset $Y$ of $X$, and a homeomorphism $h$ of $Y$ into $E$. For exch $b \in B$, let $L_{b}$ denote the linear extension of $P_{b}$; then $I_{b}$ is a closed subspace of $E$ and is itself an accessible normed linear space. For each $b$, let $S_{b}$ denote the set $h^{-1}\left(L_{b}, h Y\right)$. Then $Y=\bigcup_{b \in B} S_{b}$, each set $S_{b}$ is closed, and $b ; b^{\prime}$ implies
$S_{b} \subset S_{b^{\prime}}$. By a well-known property of separable metric spaces ([15], p. 146) there must exist a countable sequence $b_{I} ; b_{2}-\ldots$ in $B$ such that $\bigcup_{b \in B} S_{b}=\bigcup_{i=1}^{\infty} S_{b_{i}}$. But each set $S_{b_{i}}$ is of the first category in $X$ by the inductive hypothesis, so the same must be true of their union, the set $Y$. The proof is complete.

Since Hilbert space $l^{2} \kappa_{0}$ is of the second category in itself, 2.8 may be regarded as an extension of Kunugui's result [14] that $l^{2} s_{0}$ cannot be topologically embedded in $l_{F}^{2} \kappa_{0}$.

Michael considered transformations $q$ (called carriers) which map a topological space $X$ into the class of nonempty subsets of a topological space $Y$. A selection for $p$ is a continuous map $f$ of $X$ into $Y$ such that $f x \in p x$ for all $x \in X$. Michael proved ([22], [23]):

$\mathrm{M}^{0}$. If $X$ is a paracompact $T_{1}$-space and $\varphi$ is a lower semicontinuous carrier mapping $X$ into the class of nonempty closed convex subsets of a Banach space $Y$, then $\varphi$ admits a selection.

From $\mathrm{M}^{0}$ he deduced the result 2.2 above. He showed by example ([23], p. 374) that $\mathrm{M}^{0}$ may fail when $Y$ is not complete. In [24] he considered continuous carriers, and conjectured ([24], p. 389) that even when $\varphi$ is continuous, $\mathrm{M}^{0}$ may fail if $Y$ is not complete. He also conjectured that 2.2 may fail in the absence of completeness. Now of course $2.2 \mathrm{can}$ fail in a trivial way-use 2.1 to produce a binnique continuous linear map $u$ of the space $l_{f} c$ onto a separable Banach space and observe that $u^{-1}$ is discontinuous. But then $u^{-1}$ is also not a continuons carrier in Michael's sense. Now consider an arbitrary infinite-dirnensional separable Banach space $D$. By the second part of 2.1, there exists a continuous open linear transformation $u$ of $l_{F c}$ onto $E$, and the carrier $u^{-1}$ is continuous in the sense of $[24]$, p. 377 . The carrier $u^{-1}$ does not admit a continuous selection (and the transformation $u$ does not admit a continuous inverse in the sense of 2.2 ) because such a selection would be a homeomorphism of $E$ into $l_{F} c$, and by 2.8 no such homeomorphism exists.

Note that in 2.2, the transformation $u$ must be open by the open mapping theorem [1]. If it be assumed, on the other hand, that $F^{\prime}$ is a Banach space and $u$ is a continuous open linear transformation of $F$ onto the normed linear space $E$, then completeness of $E$ follows from a theorem of Ptak ([26], p. 70).

\section{References}

[1] \$. Banach, Théorie des opérations linéaires, Warszawa 1932.

[2] - and S. Mazur, Zur theorie der linearen Dimension, Studia Math. 4 (1933), p. 100-112.

[3] R. G. Bartle and L. M. Graves, Mappings between function spaces, Trans. Amer. Math. Soc. 72 (1952), p. 400-413.

Funciamenta Mathematicae, т. XIIX. 
[4] K. Borsuk, Drei Sätze über die n-dimensionale Enklidisehe sphläre, fund. Math. 20 (1933), p. 177-190.

[5] C. H. Dowker, An imbedding theorem for paracompact metric spaces, Duke Math. J. 14 (1947), p. 639.645

[6] Maurice Fréchet, Les espaces abstraits, Paris 1928.

[7] M. I. Kadeč, On topological equivalence of uniformly convex spaces, Uspeklij 11a.t. Nauk 10, pt. 4 (66) (1955), p. 137-141 (Russian).

[8] - On weak and norm eonnergence, Doklady Akad. Nauk SSSR 122 (1958), p. $13-16$ (Russian)

[9] M. Katětop, On mappings of countable spaces, Colloquium Math. 2 (1949), p. $30-33$

[10] Victor Klee, Some topological properties of convex sets, Truns. Amer. Math. Soc. $78(1955)$, p. $30-45$.

[11] - On a problem of Banach, Colloquium Math. 5 (1957), p. 78.

[12] - On the borelian and projeetive types of linear subspaces, Math. Scand. (1958), p. 189-199.

[13] - The topological structure of infinite-dimensional linear spaces, to appern.

[14] K. Kunugui, Sur un nombre infinni de dimensions inférieur à celui de l'espae de Hilbert, C. R. Acad. Sci. Paris 187 (1928), p. 876-878.

[15] C. Kuratowski, Quelques problèmes concemant les espaces metriques nonseparables, Fund. Math. 25 (1935), p. 534-545.

[16] - Topologie $I$, $4^{\text {th }}$ edition, Warszawa 1958.

[17] H. Löwig, Über die Dimension linearer Räume, Studia Math. 5 (1934), p. 18.23.

[18] G. W. Mackey, On infinile-dimensional linear spaces, Trans. Amer. Math. Soc. 57 (1945), p. $155-207$.

[19] E. Marczewski, Separabilité et multiplication carlésienne des espaces topologiques, Fund. Math. 34 (1947), p. 127-143.

[20] S. Mazur, Dne remarque sur l'lomeomorphio des ohamps fonctionnels, Stndia Math. 1 (1929), p. 83-85.

[21] Edward James MeShane, Integration, Princeton 1944.

[22] E. Michael, Seleeted selection theorems, Amer. Math. Monthly 63 (1956), p. $233-238$

[23] - Continuous selections $I$, Ann. of Math. 63 (1956), p. 361-382.

[24] - Continuous selections III, Ann. of Math. 65 (1957), p. 375-390.

[25] A. Parhomenko, Uber eineindeutige stetige Abbildungen, Rec. Math. (Mat-

Shornik) N. S. 5 (47) (1939), p. 197-210 (Russian. German summary).

[26] Vlastimil Ptak, Completeness and the open mapping theorem, Bull. Soc. Math. France 86 (1958), p. 41-74.

[27] R. Sikorski, Remark on a problem of Banach, Colloquium Math. 1 (1948), p. $285-288$.

[28] A. H. Stone, Paracompactness and product spaces, Bull. Amer, Mat]. Soc $54(1948)$, p. $977-982$

[29] Gordon Thomas Whyburn, Analytic topology, New York 1942.

THE UNIVERSITY OF COPENHAGEN AND THE UNIVERSITY OF WASHINGTON

Reģu par la Rédaction le 3.11. 1959

\section{Arithmetization of metamathematics in a general setting}

by

\section{S. Feferman* (Stanford, Calif.)}

\section{Introduction}

The method of axithmetization, as developed by Gödel [10], explojts the possibility of defining within a formal theory $\mathcal{G}$, or in arithmetical theories closely related to $\mathcal{G}$, varions syntactical and logical notions concerning $\mathcal{G}$. In broad terms, the applications of the method can be classified as being extensional if essentially only numerically correct definitions are needed, or intensional if the definitions must more fully express the notions involved, so that various of the general properties of these notions can be formally derived.

The following are some results of extensional type: incompleteness theorems (Gödel's first underivability theorem [10] Satz VI, Rosser [29] Theorem II); non-definability of predicates in formal theories (Tarski [31], Kleene [15] Theorem XIII); undecidability of various theories (Rosser [29] Theorem III, Tarski, Mostowski and Robinson [32]); and degrees of unsolvability of various theories (Myhill [25], our [7]). Among the intensional results we have the following: unprovability of consistency statements (Gödel's second underivability theorem [10] Satz XI), comparison of theories by relative consistency proofs (Novak [26], Wang [36], [37], Shoenfield [30]); and ordinal logics (Turing [33], our [8]). A result of mixed character is the arithmetization of Godel's completeness theorem

* The results reported in this paper were obtained while the author was a student of Professor Altred Tarsli at the University of California, Berkeley. A more complete presentation of them has been given in the author's thesis [4]; announcement of the results. lias also boen made in [5] and [6].

We are indehtod to Prolessor Tarski for a number of helpful suggestions regarding this resedrch; as well as to Professor Leon 'Honkin for his kind guidance during the period 1955-50 when P'rofeesor Tarski was on leave. We wish also to thank Professors John Mylill and Georg Kreisel, both for a number of stimulating conversations, and also the latter for his helpful comments on a draft of this paper.

Finally, thanks are due to Professor Steven Orey for his interest in widening the range of application of our work, as will be evidenced at various points in the text.

for the Otfice of Ordnance Research, U.S.A. 